\title{
The physical structure of the planetary nebula NGC 6781
}

\author{
F. Mavromatakis ${ }^{1}$, J. Papamastorakis ${ }^{1,2}$, and E. V. Paleologou ${ }^{2}$ \\ 1 University of Crete, Physics Department, PO Box 2208, 71003 Heraklion, Crete, Greece \\ 2 Foundation for Research and Technology-Hellas, PO Box 1527, 71110 Heraklion, Crete, Greece
}

Received 22 March 2001 / Accepted 15 May 2001

\begin{abstract}
The planetary nebula NGC 6781 was imaged in major optical emission lines. These lines allow us to construct maps of the projected, two dimensional Balmer decrement, electron density, electron temperature, ionization and abundance structure. The average electron density, determined from the [S II] lines, is $\sim 500 \mathrm{~cm}^{-3}$, while the electron temperature distribution, determined from the $[\mathrm{N} \mathrm{II}]$ lines, is flat at $\sim 10000 \mathrm{~K}$. The Balmer decrement map shows that there are variations in extinction between the north and south areas of the planetary nebula. The higher extinction observed to the north of the central star is probably caused by dust spatially associated with $\mathrm{CO}$ emission at blue-shifted velocities. The [N II] image reveals the known optical halo, at a flux level of $\sim 0.2 \%$ of the strong shell emission in the east, but now the angular extent of $216^{\prime \prime} \times 190^{\prime \prime}$ is much larger than previous measurements. The halo is also present in [O III], where we measure an extent of $190^{\prime \prime} \times 162^{\prime \prime}$. The ionization maps indicate substantial ionization along the caps of the ellipsoid as well as in the halo. The maps also show a sharp decrease in ionization along the outer edge of the shell in the west and the east, south-east. The typical log abundances measured for He, N, O and S are 10.97, 8.14, 8.72 and 6.90, respectively.
\end{abstract}

Key words. ISM: general - ISM: planetary nebulae - planetary nebulae: NGC 6781

\section{Introduction}

A planetary nebula $(\mathrm{PN})$ starts forming when a star, during its passage from the asymptotic giant branch (AGB), loses mass from its outer layers through the action of wind. Typical mass-loss rates and wind velocities are of the order of $\sim 10^{-7}-10^{-6} M_{\odot} \mathrm{yr}^{-1}$ and $\sim 10-20 \mathrm{~km} \mathrm{~s}^{-1}$, respectively, in these first stages of PN creation. In later stages of the star's evolution, higher mass-loss rates are achieved $\left(\sim 10^{-4} M_{\odot} \mathrm{yr}^{-1}\right)$ while the wind velocity may increase by a much smaller factor $(\sim 2)$. Finally, when the central star is well beyond the AGB phase and the ultraviolet photon flux is strong, material is driven away from the star at high velocities $\left(\sim 1000 \mathrm{~km} \mathrm{~s}^{-1}\right)$ and low mass-loss rates $\left(\sim 10^{-8} M_{\odot} \mathrm{yr}^{-1}\right)$. The interaction of these winds, produced in different stages of the star's evolution, defines the general framework of a difficult problem involving radiation physics and gas dynamics. Numerical models based on the interacting stellar wind scenario appear to describe sufficiently well the overall observational properties of PNe (e.g. Frank \& Mellema 1994; Corradi et al. 2000 and references therein). The models show that for typical parameters describing the central star, the appearance of a PN depends crucially upon the inclination angle and the pole-to-equator density contrast. Numerical

Send offprint requests to: F. Mavromatakis, e-mail: fotis@physics.uoc.gr simulations by Frank et al. (1993) and Zhang \& Kwok (1998) show how the optical appearance of a PN varies with these parameters at any given time. Optical observations of a large number of planetary nebulae (e.g. Balick 1987; Corradi \& Schwarz 1995) show that this class of objects possesses a wide range of different morphologies, like elliptical, bipolar, point symmetric and irregular. In addition, some of these nebulae display small-scale features known as FLIERS, tails etc. (Balick 1987; Balick et al. 1994).

The planetary nebula NGC 6781 lies $\sim 3^{\circ}$ below the galactic plane and is believed to be located at a distance of $800 \mathrm{pc}$ (Acker 1978). It is morphologically similar to NGC 6720 (the Ring Nebula), although it has a much lower surface brightness. Balick (1987) presented CCD images in the light of $\mathrm{H} \alpha$, [O III], and [N $\mathrm{II}]$ and characterized it as a middle butterfly nebula. The optical extent of the main nebula is $\sim 95^{\prime \prime}$, while the optical halo has a much larger extent, around $180^{\prime \prime} \times 109^{\prime \prime}$ (Chu et al. 1987). Górny et al. (1997) studied the properties of the central stars of 72 PNe through models of stellar evolution. The authors estimate a temperature of $\sim 10^{5} \mathrm{~K}$, a luminosity of $\sim 200 L_{\odot}$ and an evolutionary time scale of $\sim 17000$ yr for the central star in NGC 6781. Zuckerman et al. (1990) observed this PN in $\mathrm{H}_{2}$ and $\mathrm{CO}$ and detected extended emission, suggesting that it contains a significant amount of mass in molecular form. Subsequent, high 
resolution CO observations by Bachiller et al. (1993) revealed more details about the distribution and the kinematics of the molecular gas. In the light of their observations, the authors proposed a model consisting of a thin ellipsoidal shell, truncated at the two ends. They found that the major axis of the prolate ellipsoid is inclined by $\sim 23^{\circ}$ with respect to the line of sight and that the model could account for some of the observed properties.

In this work we present deep, flux calibrated CCD images of NGC 6781 in main optical emission lines. The new optical images reveal more details about the morphology of the nebula than were previously known and allow us to construct maps of the projected two dimensional distribution of the electron temperature and the electron density. In addition, these images can be used to study the ionization state and structure of the nebula, to search for possible abundance variations and also, for small scale structures. In Sect. 2 information about the observations is given, while in Sect. 3 we present the results of the image processing on the morphology of the nebula and the electron density and temperature distribution. Finally, in Sect. 4 we discuss the implications of our imaging observations on our current knowledge of the physical properties of NGC 6781.

\section{Observations}

\subsection{Optical images}

The planetary nebula NGC 6781 was observed with the 1.3 $\mathrm{m}$ telescope at Skinakas Observatory. The $f / 7.7$ Ritchey - Cretien telescope was used in connection with a focal reducer, resulting in a field of view enlarged by a factor of 1.88 . We note here that the filters were placed in the optical path before the focal reducer and that the images were $2 \times 2$ binned during the CCD readout process to increase the signal to noise ratio. The observations were performed with a $1024 \times 1024$ Thomson CCD having $19 \mu \mathrm{m}$ square pixels resulting in a $12^{\prime} \times 12^{\prime}$ field of view. The pixel size after binning was 1 ". 5 . The [O II] and He II observations were performed with a $1024 \times 1024$ Site CCD, having $24 \mu \mathrm{m}$ square pixels, because it had a better response in this wavelength band. The filters used during the observations, their characteristics and the relevant exposure times are listed in Table 1 . The $\mathrm{H} \alpha$ and [N II] filters are contaminated by the $6548 \AA$ line of nitrogen and the $6563 \AA$ line of hydrogen, respectively. The filter transmission curves were used to separate individual lines and a ratio of $I(6584) / I(6548)$ of 3 was adopted. Also, the $[\mathrm{S} \mathrm{II}]_{2}$ is contaminated by the $6716 \AA$ line and the filter transmission curves of both $[\mathrm{SII}]_{1}$ and $[\mathrm{S} \mathrm{II}]_{2}$ were used to separate theses lines. Standard IRAF and MIDAS routines were used for the reduction of the data. Individual frames were bias subtracted and flat-field corrected using a series of well exposed twilight flat-fields. The data were flux calibrated using the spectrophotometric standard stars HR 7596, HR 9087, HR 8634 and HR 5501.
Table 1. Observational log and filter characteristics.

\begin{tabular}{lllll}
\hline Filter & $\begin{array}{l}C W L^{\mathrm{a}} \\
(\AA)\end{array}$ & $\begin{array}{l}F W H M^{\mathrm{b}} \\
(\AA)\end{array}$ & $\begin{array}{l}\text { Exposure }^{\mathrm{c}} \\
\text { time}(\#)^{\mathrm{d}}\end{array}$ & Date \\
\hline$[\mathrm{O}$ II $]$ & 3727 & 20 & $9000(5)$ & $10-06-1999$ \\
\hline $\mathrm{He}$ II & 4687 & 20 & $14400(8)$ & $10-06-1999$ \\
\hline $\mathrm{H}_{\beta}$ & 4861 & 20 & $5400(3)$ & $01-07-1997$ \\
\hline$[\mathrm{O}$ III $]$ & 5005 & 20 & $5400(3)$ & $30-06-1997$ \\
\hline$[\mathrm{N} \mathrm{II}]$ & 5755 & 12 & $21600(12)$ & $02,03-07-1997$ \\
\hline $\mathrm{He} \mathrm{I}$ & 5876 & 20 & $10800(6)$ & $11-06-1999$ \\
\hline $\mathrm{H} \alpha$ & 6555 & 12 & $5400(3)$ & $30-06-1997$ \\
\hline$[\mathrm{N}$ II $]$ & 6584 & 20 & $3600(2)$ & $30-06-1997$ \\
\hline$[\mathrm{S} \mathrm{II}]_{1}$ & 6716 & 12 & $7200(4)$ & $02,03-06-1997$ \\
\hline$[\mathrm{S} \mathrm{II}]_{2}$ & 6731 & 12 & $7200(4)$ & $02,03-06-1997$ \\
\hline Contred & 6096 & 134 & $480(4)$ & $01,02-06-1997$ \\
\hline Contblue & 5470 & 240 & $480(4)$ & $10,11-6-1999$ \\
\hline
\end{tabular}

${ }^{a}$ Wavelength at peak transmittance for $f / 7.7$.

${ }^{\mathrm{b}}$ Full width at half maximum.

c Total exposure time in s.

d Number of exposures, each of $1800 \mathrm{~s}$ duration.

\section{Results}

\subsection{Morphology}

The morphology of the planetary nebula NGC 6781 depends on the line imaged (Fig. 1). The images taken at

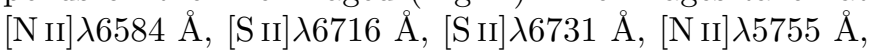
He I $\lambda 5876 \AA$ and $[\mathrm{O}$ II $] \lambda 3727 \AA$ display the known "C"-like structure. This structure is characterized by an annulus which is open in the north, north-west. It is roughly defined by two circles with radii of $38^{\prime \prime}$ and $60^{\prime \prime}$, respectively, centered on the central star. The annulus defines the shell area of the nebula while the inner circular area (radius $\left.<38^{\prime \prime}\right)$ defines the core. The brightest parts of the observed emission are found inside the shell and at opposite sides, lying roughly in the east - west direction. The flux inside the missing part of the shell in the north is less, by a factor of $\sim 2.8$, than the flux measured in the bright area in the east, inside the shell. This conclusion holds for all of the above mentioned line images. Emission outside the largest ring is seen in the $[\mathrm{O}$ II $] \lambda 3727 \AA$ and $\left[\mathrm{N}_{\mathrm{II}}\right] \lambda 6584 \AA$ low ionization lines as well as in the $[\mathrm{O}$ III $] \lambda 5007 \AA$ higher ionization line. Particularly, in the $\left[\mathrm{N}_{\mathrm{II}}\right]$ image, faint diffuse emission (the halo) is detected at larger angular distances than was known before. A circular area, centered on the central star, with a radius of $\sim 120^{\prime \prime}$ seems to encompass all of the $\left[\mathrm{N}_{\mathrm{II}}\right] \lambda 6584 \AA$ emission (Fig. 2). Fitting an ellipse to the $4 \sigma$ isophotes, we find a nebular extent along the major axis of $216^{\prime \prime}$ which is inclined to the west by $23^{\circ}$ and an extent along the minor axis of $190^{\prime \prime}$. At the same level of confidence, the [O III] image suggests an extent along the major axis of $190^{\prime \prime}$ inclined by $19^{\circ}$ to the west, and an extent along the minor axis of $162^{\prime \prime}$. These values 

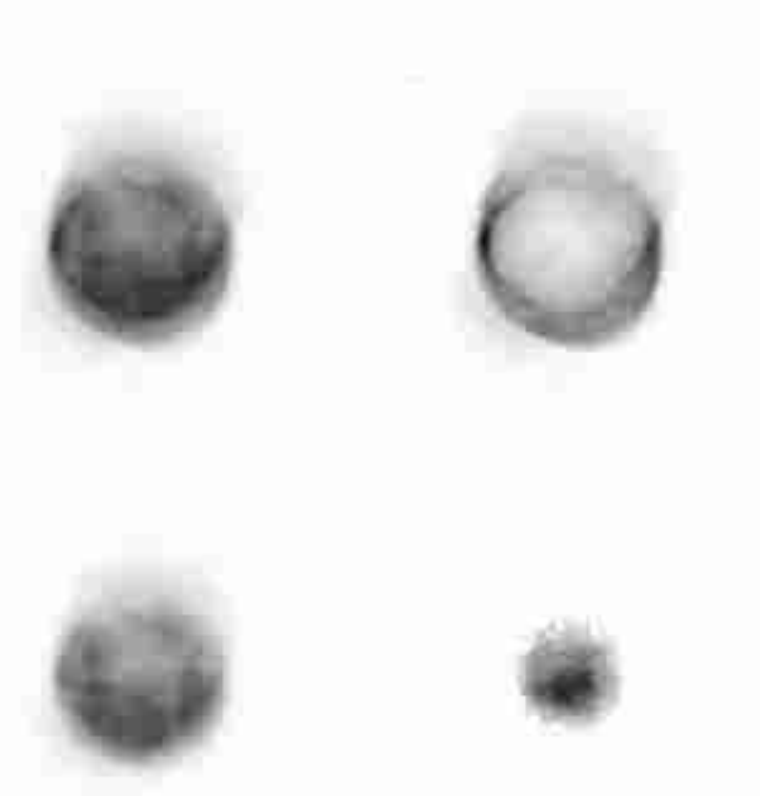

Fig. 1. NGC 6781 imaged in $\mathrm{H} \alpha$ (top left), [N II]6584 $\AA$ (top right), [O III] $5007 \AA$ (bottom left) and He II $4686 \AA$ (bottom right). Here and in all subsequent figures east is to the left and north to the top.

are significanlty larger than the dimensions of $180^{\prime \prime} \times 109^{\prime \prime}$ given by Chu et al. (1987).

The image of the nebula as seen in the HeII 4686 line differs from the images in all other lines observed. The bulk of the twice ionized helium emission is confined by the inner circle of $\sim 38^{\prime \prime}$ radius, at the $4 \sigma$ limit, and has a round shape. The central star is found at the center of symmetry of this round shape. Interestingly, the morphology of the strongest He II emission $(\sim 30-40 \times$ $10^{-17} \mathrm{erg} \mathrm{s}^{-1} \mathrm{~cm}^{-2} \operatorname{arcsec}^{-2}$ ) displays a "drop-like" shape. The central star is not at the center of this "structure" but lies at its north boundary. On the other hand, the images from $\mathrm{H} \alpha, \mathrm{H} \beta$ and $[\mathrm{O}$ III] $\lambda 5007 \AA$ show a different morphology where strong emission is found both in the core and the shell but also outside the annulus. There is no distinct boundary between the shell and the core but rather a smooth distribution of the flux. All three images reveal a deficiency in emission to the north of the central star. Faint diffuse emission is detected in the south-east and north-west out to distances of $\sim 90^{\prime \prime}$ from the center.

\subsection{The Balmer decrement}

The ratio image of $\mathrm{H} \alpha / \mathrm{H} \beta$ shows that the extinction is not constant over the nebula. The mean value of the Balmer decrement inside the bright shell, shown in Fig. 3 (top), is $5.6( \pm 0.2)$ to the south of the central star, while to the north reaches values of $7.0( \pm 0.5)$. It is known that the Balmer decrement depends slightly on the temperature

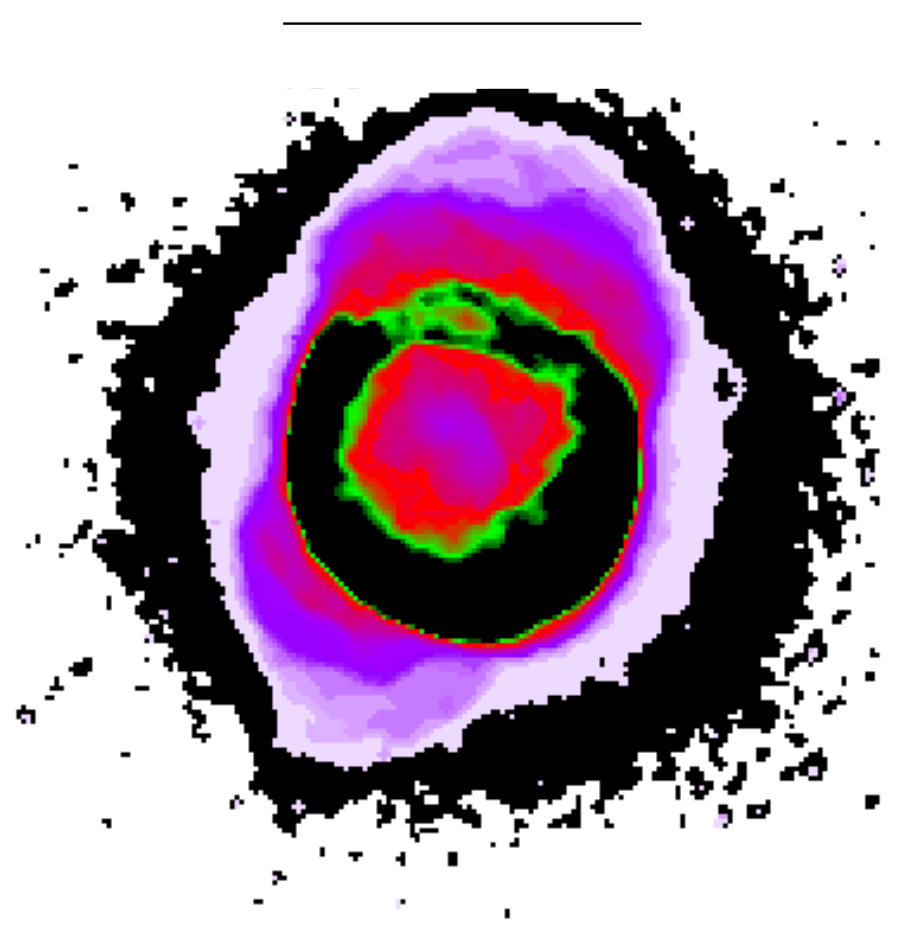

Fig. 2. The halo of NGC 6781 imaged in the light of the [N II] $6584 \AA$ line. The halo emission is quite weak and is shown with the outer black colour. The image has been clipped at the $4 \sigma$ level.

(e.g. Osterbrock 1989) but the calculations of Hummer \& Storey (1987) show that it is not expected to vary by more than a few percent given the physical conditions found in this nebula (this work, Kaler et al. 1990). The difference here is $\sim 25 \%$. In order to obtain better estimates of the decrement outside the bright shell, we average the $\mathrm{H} \alpha$ and $\mathrm{H} \beta$ fluxes over a sufficient number of halo pixels. The mean value measured in the south is $5.9( \pm 0.5)$, and $6.5( \pm 0.3)$ in the north. These results agree, roughly, within $1 \sigma$ and thus, it is not possible to state confidently variations between the south and north outer areas, in contrast to the variations between the corresponding inner regions, which are most likely true. The $\mathrm{H} \alpha / \mathrm{H} \beta$ image and the interstellar extinction law of Cardelli et al. (1989) were used to construct an extinction map under the assumption of an intrinsic Case B recombination ratio of 2.86 (Hummer \& Storey 1987). This extinction map has been used in our work to correct all flux images for the extinction.

\subsection{The electron density}

The line images of $\left[\mathrm{S}_{\mathrm{II}}\right] \lambda 6716 \AA$ and $[\mathrm{S}$ II $] \lambda 6731 \AA$ allow us to study the projected two dimensional structure of the electron density (Fig. 3 bottom). The ratio of the intensities of these two lines depends mostly on the electron density of the emitting element and much less on the temperature (e.g. Aller 1984). Kaler et al. (1990) measured, through low ionization lines, electron temperatures around 9000-10000 K at two different locations in the nebula. 


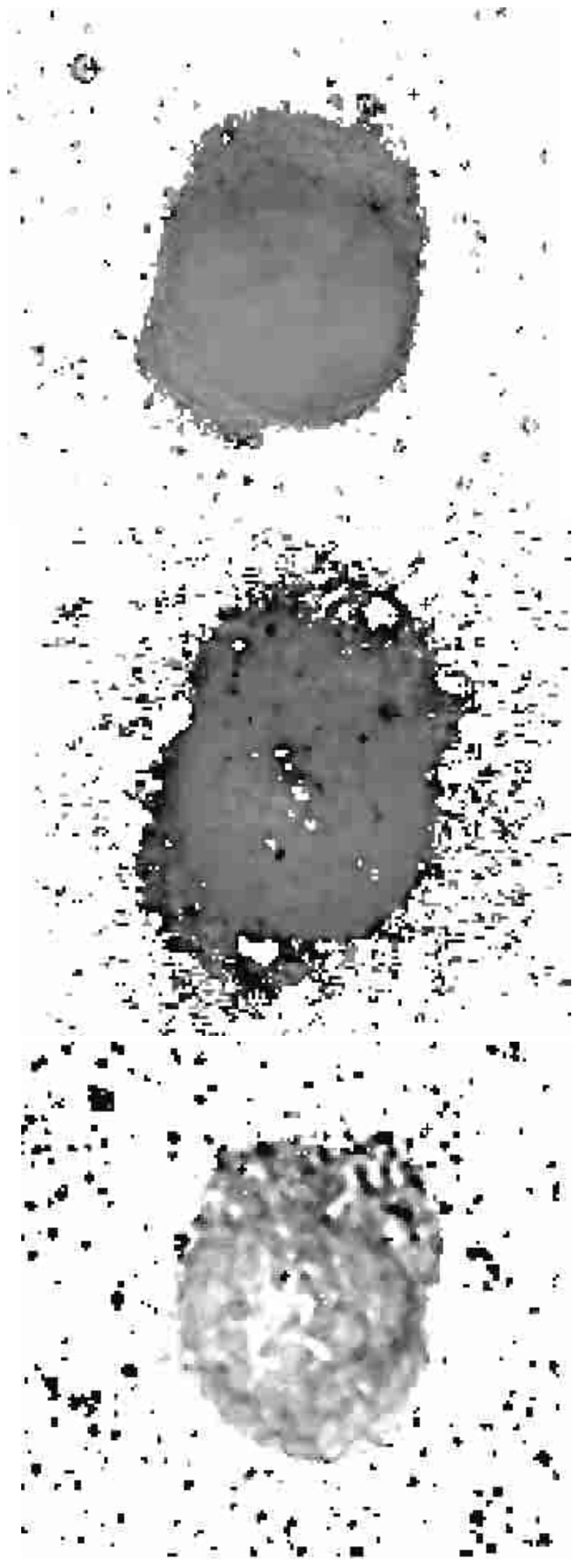

Fig. 3. The spatial distribution of the Balmer decrement clipped at the level of $4 \sigma$ (top). The middle image shows the spatial distribution of the electron temperature in NGC 6781 where typical temperatures lie in the range of 9000-10 $500 \mathrm{~K}$. The spatial distribution of the electron density clipped at a level of $4 \sigma$ is shown in the bottom image. The measured electron densities are found in the range of $400-600 \mathrm{~cm}^{-3}$.
The authors also used higher ionization lines to measure temperatures up to $15000 \mathrm{~K}$ in the same locations. Even for this range of temperatures, the derived electron densities do not vary significantly. The conversion from the sulfur line ratio to electron density utilized the analytical formulas provided by Alexander \& Balick (1997) assuming an electron temperature of $10000 \mathrm{~K}$ (see also Sect. 3.4). The observed fluxes of the sulfur lines are characterized by a signal to noise per pixel in the range of 20-30 and 10-16 in the shell and the core of the nebula, respectively. The resulting image shows that there are variations in the electron density across the nebula. The average electron density in the south, south-east is $\sim 440 \mathrm{~cm}^{-3}$ with an rms scatter of $100 \mathrm{~cm}^{-3}$, while in the north-west part of the shell, densities of $\sim 600( \pm 100) \mathrm{cm}^{-3}$ are found.

\subsection{The electron temperature}

The electron temperature in low ionization regions was estimated through the line ratio involving the nitrogen lines at $6548 \AA, 6583 \AA$ and $5755 \AA$. Again the analytical formula provided by Alexander \& Balick (1997) was used for an assumed electron density of $400 \mathrm{~cm}^{-3}$. The electron temperature image (Fig. 3 middle) does not show substantial variations in the shell area while it seems that there is a minor temperature increase towards the core area. We find typical shell temperatures $\sim 9000 \mathrm{~K}$ with an rms scatter of $100 \mathrm{~K}$. Slightly higher temperatures are found in the core $\sim 10500 \mathrm{~K}$ but the rms scatter also increases to $500 \mathrm{~K}$. These temperatures are in agreement with those of 9000-10400 K measured by Kaler et al. (1990) using the same diagnostic lines.

\subsection{The ionization state}

The ratio images of $[\mathrm{N}$ II $] / \mathrm{H} \alpha$ (Fig. 4a), $\mathrm{H} \alpha+[\mathrm{N}$ II $] /[\mathrm{O}$ III $]$, $[\mathrm{O}$ II $] /[\mathrm{O}$ III $], \quad \mathrm{H} \alpha /[\mathrm{S} \mathrm{II}], \quad[\mathrm{N} \mathrm{II}] /[\mathrm{O}$ III $], \quad[\mathrm{O}$ III $] / \mathrm{H} \beta \quad$ and $\mathrm{He} \mathrm{II} / \mathrm{H} \beta$, which are sensitive to the ionization structure and ionizing mechanism, were calculated. Some of these line ratio images, masked at the $4 \sigma$ confidence level, are shown in Fig. 4.

The $[\mathrm{N} \mathrm{II}] \lambda 6584 \AA /[\mathrm{O}$ III $]$ image (Fig. 4c) displays its lowest values in the core area suggesting the high degree of ionization there, in agreement with the $\mathrm{He}$ II/ $\mathrm{H} \beta$ image map. The ionization stage decreases rapidly from the inner to the outer shell boundary (values increase from $\sim 0.5$ to $\sim 3.0$ ) mainly in the east and west, south-west areas of the shell. In the north part of the shell we observe a gap in the low ionization front seen previously (valus increase only up to $\sim 0.8$ ) which seems to be connected to the gap seen in the intensity images (Fig. 1), while in the south-east part of the shell a smaller gap than in the north is present which shows moderate ionization $([\mathrm{N} \mathrm{II}] /[\mathrm{O}$ III $]$ values up to 1.2 ). The emission outside the shell, in the south-east and north-west, is found in an ionization state similar to that of the shell. The morphology of the [O II]/[O III] image is identical to that of the $[\mathrm{N} \mathrm{II}] /[\mathrm{O} \mathrm{III}]$ (Fig. 4c) image 

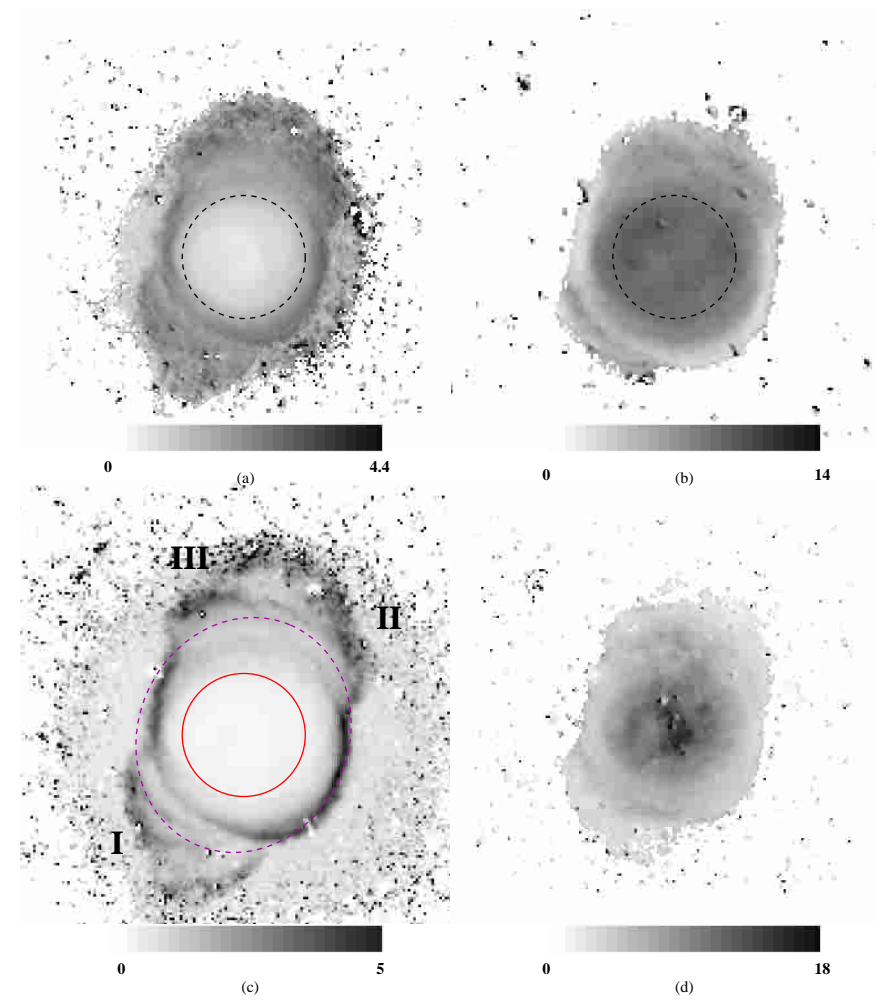

Fig. 4. The diagnostic ratios of $[\mathrm{N} \mathrm{II}] / \mathrm{H} \alpha$ (top-left), $[\mathrm{O}$ III $] / \mathrm{H} \beta$ (top-right), [N II]/[O III] (bottom-left) and $\mathrm{H} \alpha /[\mathrm{S} \mathrm{II}]$ (bottomright) are shown in linear grey scale (minima $\rightarrow$ white, maxima $\rightarrow$ black). All images are clipped at the $4 \sigma$ level. The circle defines the core area, is centered on the central star and has a radius of $38^{\prime \prime}$. The dashed ellipse respresents the outer projected boundary of Bachiller's et al. (1993) ellipsoid.

since low ionization lines ([N II $],[\mathrm{O}$ II $]$ ) are compared to the same moderate ionization line $([\mathrm{O} \mathrm{III}])$. The $[\mathrm{N} \mathrm{II}] /[\mathrm{O}$ III $]$ and $[\mathrm{N} \mathrm{II}] / \mathrm{H} \alpha$ image maps do not provide evidence for low ionization, small scale structures (Corradi et al. 1996).

The $[\mathrm{O}$ III $] / \mathrm{H} \beta$ (Fig. 4b) image shows constant ionization in the core but variations are observed in the shell. A mean value of 8.5 with an rms of 0.5 is measured inside a radius of $45^{\prime \prime}$ but outside this radius, values in the range of 2.5 to 7 are measured. The [O III] ionization front is rather broad, $\sim 20^{\prime \prime}\left(\sim 2 \times 10^{17} \mathrm{~cm}\right)$, and its shape shows partial circular symmetry. The $[\mathrm{O}$ III $] / \mathrm{H} \beta$ map clearly displays strong ionization extending out of the shell in the north-west and south-east directions, while it does not show any evidence for a strong $\mathrm{O}^{+3}$ ionization front. The $\mathrm{He} \mathrm{II} / \mathrm{H} \beta$ image shows that the He II ionization front is roughly circular with a radius of $\sim 38^{\prime \prime}$.

The $\mathrm{H} \alpha /[\mathrm{S} \mathrm{II}]$ (Fig. 4d) image indicates that the dominant excitation mechanism in the core area is photoionization (values $>5$ ) whereas values below 4.5 are measured in the shell. Especially, in the east and west areas outside the shell the $\mathrm{H} \alpha /[\mathrm{S}$ II] ratio drops to less than 2.9 and might indicate the presence of shocked ionized material (e.g. Bohigas 1994).

\subsection{Abundances}

The interference filters used to image NGC 6781 allow us to study the spatial distribution of the ionic abundances relative to $\mathrm{H}^{+}$as well as the total abundances of helium, nitrogen, oxygen and sulfur. In order to perform these calculations we used the algebraic expressions given by Alexander \& Balick (1997) both for the ionic abundances relative to hydrogen and the ionization correction factors. The helium lines of $4686 \AA$ and $5876 \AA$ are used to calculate an average helium abundance of $0.093( \pm 0.006)$. The equivalent log abundance ratio is 10.97:12.00 with an error of \pm 0.04 .

The two-dimensional image of the nitrogen abundance shows a slight stratification from south to north. We measure an average abundance of $1.4( \pm 0.1) \times 10^{-4}$ in the south, an abundance of $1.6( \pm 0.2) \times 10^{-4}$ in the central area of the nebula and an abundance of $1.3( \pm 0.1) \times 10^{-4}$ in the north part of the shell. Formally, these variations are not statistically significant but the trend seen in the relevant image is, probably, true. The weighted average $\log$ abundance of these values is 8.14:12.00 with an error of 0.04 .

The oxygen abundance is roughly constant at an average value of $5.3( \pm 0.5) \times 10^{-4}$, or equivalently at a $\log$ abundance ratio of $8.72: 12.00$ with an error of 0.06 . However, we note here that there is an increase in the oxygen abundance to $\sim 7.5( \pm 0.6) \times 10^{-4}$ close to the west, outer boundary of the shell.

The morphology of the sulfur abundance map is similar to that seen in the related flux images, i.e. enhanced values in the shell and reduced in the core. We measure an average abundance of $8.5( \pm 0.7) \times 10^{-6}$ in the south, south-east part of the shell while an abundance of $10( \pm 0.7) \times 10^{-6}$ is found in the west part of the shell, in the same region where we observe an enhancement in the oxygen abundance. This is not surprising since Alexander \& Balick (1997), in a series of model nebulae, demonstrated the presence of a zone of neutral hydrogen where $\mathrm{S}^{+}$ions are still present. The sulfur abundance in the core area reduces to $5.3( \pm 0.7) \times 10^{-6}$. The overall average log abundance ratio of sulfur is $6.90: 12.00$ with an error of 0.06 .

\subsection{Integrated properties of NGC 6781}

The total flux of the $\mathrm{H} \beta$ is listed in Table 2 along with basic line ratios. The surface brightness of each line was integrated over an area of $118^{\prime \prime} \times 124^{\prime \prime}$, along right ascension and declination, respectively. This rectangular aperture was centered on the central star. In case of line ratios we calculate the numenators and denominators separately and then form the ratio. There is only one measurement of the $\mathrm{H} \beta$ flux available in the literature by Collins et al. (1961). However, we cannot compare our value with that of $7.4 \times 10^{-11} \mathrm{erg}^{-1} \mathrm{~s}^{-1} \mathrm{~cm}^{-2}$ given by Collins et al. (1961), mainly due to the lack of knowledge of the actual aperture used. 
Table 2. Integrated properties of NGC 6781.

\begin{tabular}{ll}
\hline $\mathrm{H} \beta^{\mathrm{a}}$ & $1.5 \times 10^{-11} \mathrm{erg} \mathrm{s}^{-1} \mathrm{~cm}^{-2}$ \\
\hline $\mathrm{H} \beta^{\mathrm{b}}$ & $16.0 \times 10^{-11} \mathrm{erg} \mathrm{s}^{-1} \mathrm{~cm}^{-2}$ \\
\hline $\mathrm{H} \alpha / \mathrm{H} \beta$ & 6.4 \\
\hline $\mathrm{HeII} / \mathrm{H} \beta$ & 0.04 \\
\hline$[\mathrm{S} \mathrm{II}]_{1} /[\mathrm{S} \mathrm{II}]_{2}$ & 1.05 \\
\hline
\end{tabular}

a Observed $\mathrm{H} \beta$ flux.

${ }^{\text {b }} \mathrm{H} \beta$ flux corrected for interstellar extinction.

\section{Discussion}

Deep exposures of the planetary nebula NGC 6781 were taken in a number of important optical emission lines. The images obtained provide us with new information about the morphology of the nebula, the halo, the ionization structure, the elemental abundances, the electron density and two-dimensional structure of the temperature.

\subsection{The evolution of NGC 6781}

Measurements of the central star properties in NGC 6781 suggest a temperature of $\sim 100000 \mathrm{~K}$ and a luminosity of $\sim 200 L_{\odot}$ indicative of an evolved system according to the evolutionary tracks of Gathier \& Pottasch (1989) and Górny et al. (1997). This implies that this planetary has most likely, passed, the turn-around point and is in the stage where the luminosity of the central star continously decreases until it fades as a white-dwarf. Consequently, models in a similar evolutionary stage should be used to compare the observed properties. Corradi et al. (2000), using a radiation hydrodynamics code in spherical geometry, produced model nebulae at different evolutionary times and we use these models to compare our observations.

The surface brightness profiles along the minor axis (Fig. 5) display a significant degree of symmetry, while the major axis reveal the asymmetry due to the complicated ellipsoidal structure. Especially, the [N II] and [O III] profiles along the minor axis clearly show an offset of $\sim 10^{\prime \prime}\left(\sim 10^{17} \mathrm{~cm}\right.$ at $\left.800 \mathrm{pc}\right)$ in their peak intensities, similar to the effect seen in the low excitation models of Corradi et al. (2000, their Fig. 6), corresponding to late post-AGB ages. The major axis profiles stress the known "C" like shape of the nebula and at large angular distances from the central star, emission is detected at a level of a few percent, mainly in the low ionization lines. Provided that this emission actually originates from a "recombining halo", together with the absence of strong evidence for limb brightening, may point towards more evolved models where the edge of the "recombined halo" is less pronounced (Corradi et al. 2000, their Figs. 7-8). However, it is clear that the comparison is restricted by the lack of kinematic data and the uncertainties in the distance and the central star properties. In addition, we note that Górny et al. (1997) estimate a mass of $0.57 M_{\odot}$ and an age of $\sim 17000$ yrs following the formation of the
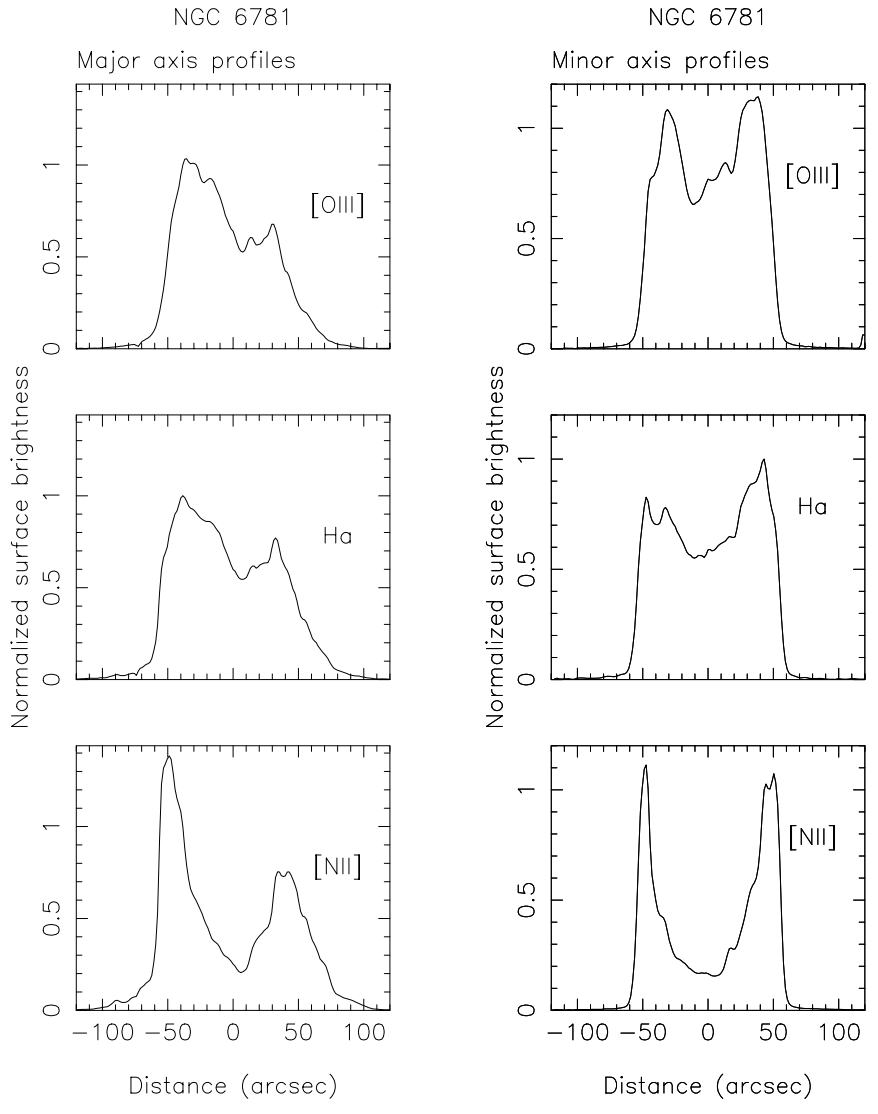

Fig. 5. Normalized surface brightness profiles along the major (left) and along the minor axis (right) of the nebula in [OIII] (top), $\mathrm{H} \alpha$ (middle) and [N II] (bottom). The profiles are the average of $10^{\prime \prime}$ wide strips through the central star and the angular distance is measured from it.

planetary nebula, whereas Gathier and Pottasch's (1989) evolutionary tracks suggest a mass of $\sim 0.546 M_{\odot}$ and a time interval of 320000-400000 yrs since the temperature of the star was $10^{3.7} \mathrm{~K}$. Their results also show that the evolutionary track of a star with the slightly higher mass of $0.565 M_{\odot}$ at $\sim 25000-40000$ yrs after its temperature was $10^{3.7} \mathrm{~K}$, is relatively close to current estimates of the central star's temperature and luminosity. It is evident that small changes in the mass of the central star leads to significant variations in the evolutionary times. Consequently, in order to understand in detail the evolution of NGC 6781, models based on the poperties of the specific system are desirable.

\subsection{The halo}

Early in the history of a PN, mass-loss episodes related to the central star evolution lead to the formation of an extended, low surface brightness "halo". The halo of NGC 6781 is characterized by a radius of $\sim 0.4 \mathrm{pc}$ at $800 \mathrm{pc}$ and its brightness in [N II] $\lambda 6584 \AA$ is $\sim 0.2 \%$ of the strong emission in the east part of the shell. The image map of $[\mathrm{N} \mathrm{II}] \lambda 6584 \AA /[\mathrm{O}$ III] (Fig. 4c) shows that the highest degree of ionization is found in the core and 
decreases radially outwards (values increase) until the outer shell boundary. The degree of ionization becomes minimum within an incomplete annulus of typical width $\sim 13^{\prime \prime}$, while outside the shell in the extended halo it increases (values decrease). The typical values of the $[\mathrm{N} \mathrm{II}] /[\mathrm{O}$ III $]$ ratio measured in the halo are only slightly higher than the values measured in the inner areas of the shell, close to the boundary with the core. This implies that electromagnetic radiation is able to penetrate the ellipsoidal structure, interact with the slow red giant wind and ionize it. Note here that the semimajor-axis extent measured in the [N II] image of $110^{\prime \prime}$ is very close to the value of $117^{\prime \prime}$ proposed by Bachiller et al. (1993) for the semimajor axis of the truncated ellipsoid.

Bryce et al. (1994) measured a ratio of $[\mathrm{N}$ II $] \lambda 6584 \AA / \mathrm{H} \alpha \simeq 1.5$ at the outer edge of the inner halo in the Ring nebula while we measure values of $\sim 1.9$ ( \pm 0.1$)$ for NGC 6781 . They attributed the increased $[\mathrm{N} \mathrm{II}] / \mathrm{H} \alpha$ ratio to a slow shock and the higher-than-solar abundance of nitrogen. These conditions may also apply to NGC 6781 since we find that the typical log abundance ratio of $\mathrm{N}: \mathrm{H}$ is $\sim 8.10: 12.00$ in the outer parts of the "C" shaped shell. This is also supported by the $\mathrm{H} \alpha /[\mathrm{S}$ II $]$ ratio which reaches its minimum value of $\sim 3.0$. This ratio can get as high as $\sim 10.0$ in the strongly photoionized regions. Finally, the estimate of the age of the halo is $\sim 41000 \mathrm{yrs}$ assuming a constant wind velocity of $10 \mathrm{~km} \mathrm{~s}^{-1}$ (e.g. Steffen et al. 1998), a distance of 800 pc and the measured angular radius of $\sim 108^{\prime \prime}$.

\subsection{The ionization and abundance structure of NGC 6781}

All ionization maps indicate the higher ionization state of the core, as expected, relative to its surroundings (Fig. 4a). The flux images of, e.g., [N II], [S II] and $\mathrm{H} \alpha$ show a decrease in flux to the north of the central star, while the ionization map of [N II]/[O III] does not exhibit a similar behavior within the core and shell areas. Instead, the degree of ionization displays an azimuthal symmetry out to $60^{\prime \prime}$ from the center with the exception of the two ionization fronts (see Sect. 3.5). Thus, the observed degree of ionization does not seem to be affected by the screening material. The carbon monoxide emission, at blue-shifted velocities (Fig. 3 of Bachiller et al. 1993), detected at small angular distances from the central star, implies that this molecular mass is located on the near side of the ellipsoid. The CO kinematic data show a gap in the $7.5 \mathrm{~km} \mathrm{~s}^{-1}$ map matching very well the gap seen in the $[\mathrm{N} \mathrm{II}] /[\mathrm{O}$ III $]$ map in the south, between the two ionization fronts. The morphology of this ratio image is quite similar to the morphology of the $\mathrm{H}_{2}$ emission from NGC 6781 (Zuckerman et al. 1990). The authors reported that the brightest molecular hydrogen emission is slightly offset with respect to the brightest $[\mathrm{N} \mathrm{II}] \lambda 6584 \AA$ emission in a way similar to what we observe. The $\mathrm{H}_{2}$ gap in the south-east as well as in the north is also reflected in the ionization map of [N II $] /[\mathrm{O} \mathrm{III}]$.
We propose that dust spatially associated with the CO emission attenuates the optical emission and causes the observed $\mathrm{H} \alpha / \mathrm{H} \beta$ morphology. The reduction in ionization in the outer part of the shell is related to the presence of $\mathrm{H}_{2}$ in these areas. An ionization edge is clearly seen in optical images of NGC 6720 where molecular hydrogen emission is also detected (Lame \& Pogge 1994).

The abundance images of helium, nitrogen, sulfur and oxygen do not reveal variations, keeping into account the simplifications made in their derivation and the statistical uncertainties, between the core and shell areas.

\subsection{The geometry of the nebula}

Kinematic studies of CO emission led Bachiller et al. (1993) to propose an ellipsoidal model for this planetary nebula. The geometrical elements of the thin, truncated ellipsoid are a semi-major axis of $117^{\prime \prime}$, a semi-minor axis of $53^{\prime \prime}$ and an inclination angle of $23^{\circ}$.

The symmetry of the outer areas of [N II] $\lambda 6584 \AA$ emission and their extent suggest that our line of sight lies close to the major axis and that the semi-minor axis extends for, at least, $60^{\prime \prime}$. Emission outside the $60^{\prime \prime}$ shell and out to $95^{\prime \prime}$ is detected in the south-east and north, north-west. Especially the emission from these regions seems to be symmetric with respect to the central star. The symmetry is more prominent in the ionization map of [N II]/[O III] (areas I, II, and III in Fig. 4c) than in the flux map of e.g. the $[\mathrm{N}$ II] line (Fig. 1). Major characteristics of this extended component are its strong flux $(\sim 15-40$ times the average halo emission), the low degree of ionization, and the probable change of the ionizing source (Fig. 4d). The [S II] $/ \mathrm{H} \alpha$ map shows that the contribution of photoionization to the gas excitation is substantially reduced compared to the core and the shell. Typically, the $[\mathrm{S} \mathrm{II}] / \mathrm{H} \alpha$ ratio is 0.10 in the core, $0.19( \pm 0.02)$ in the shell and $0.34( \pm 0.05)$ outside the shell. In addition, the temperature map does not indicate any significant variations over these regions. The patchy emission located $24^{\prime \prime}$ east and $75^{\prime \prime}$ north of the central star (area III in Fig. 4c) seems to be correlated with $\mathrm{CO}$ emission at redshifted velocities of 27.5 and $37.5 \mathrm{~km} \mathrm{~s}^{-1}$ (Bachiller et al. 1993) and could account for the reduced ionization. However, the other two patches do not have CO counterparts.

In order to explain the presence of emission outside the projected ellipsoid of Bachiller et al. (1993), we consider three possible scenarios. The first involves an ellipsoid with a semi-minor axis of $\sim 60^{\prime \prime}$, an inclination angle of $23^{\circ}$ and a semi-major axis of $\sim 200^{\prime \prime}$. This extent of the semi-major axis is required, for the given inclination angle, to project to $\sim 95^{\prime \prime}$. The second scenario does not affect the extents of the axes but the inclination angle must increase to $\sim 38^{\circ}$, to account for the emission seen $\sim 95^{\prime \prime}$ away from the central star. The third scenario does not alter the geometry of the ellipsoidal model but proposes that we may be observing the results of photoionization and wind interaction with the outer shell 
(Corradi et al. 2000). The first scenario preserves the projected circular symmetry of the waist of the ellipsoid, since the inclination is not altered but the semi-major axis is $\sim 3.3$ times greater than the semi-minor axis, implying an ellipticity of $\sim 0.95$. Even though we cannot exclude this possibility, it may be difficult to account for the processes which led to such an extreme geometry. We also note that the $\mathrm{CO}$ kinematic data do not favor such a high axial ratio. The second alternative requires only a change in the viewing angle from $\sim 23^{\circ}$ to $\sim 38^{\circ}$. However, a larger inclination leads also to an increase of the apparent ellipticity of the waist of the ellipsoid, while the low ionization lines display a circular symmetry. Finally, the third scenario suggests that we may have detected emission from a "recombining halo" (Tylenda 1986). In this case, the outer shell is expanding with a velocity higher than $\sim 30 \mathrm{~km} \mathrm{~s}^{-1}$ and its interaction with the radiation field of the central star and the AGB wind gives rise to the observed emission. Asymmetries in the mass outflow and/or AGB wind are probably present since this extended component is observed only in the south and north, north-west. A "recombining halo" is also present in NGC 2438 (Corradi et al. 2000), a system in a similar evolutionary stage as NGC 6781. Kinematic observations will provide a better understanding of the nature of the extended component and discriminate among the possible scenarios.

\section{Conclusions}

The planetary nebula NGC 6781 was mapped in several optical emission lines. The low ionization lines display the known "C" like structure. The [N II] image shows, in addition, a rather extended halo. The halo is also present in the moderate ionization line of [O III], although at a smaller extent, suggesting a relatively high ionization state. An age of $\sim 40000$ yr is estimated for a constant wind velocity of $\sim 10 \mathrm{~km} \mathrm{~s}^{-1}$. The electron temperature map does not show any strong variations and we measure an average temperature of $\sim 9000 \mathrm{~K}$. The average electron density is $\sim 500 \mathrm{~cm}^{-3}$ but variations are observed. Higher resolution observations would be required to study in detail these variations. The Balmer decrement map as well as the ionization maps indicate that dust, correlated to $\mathrm{CO}$ emission, is mainly present outside the ellipsoidal structure. The optical data favor the ellipsoidal model of Bachiller et al. (1993), while the extended emission detected outside the projected ellipsoid may represent a "recombining halo".

Acknowledgements. The authors would like to thank R. Gruenwald, B. Balick and N. D. Kylafis for their useful comments. We would also like to thank the referee R. L. M. Corradi whose pertinent remarks helped clarify, and enhance, the scope of this paper. Skinakas Observatory is a collaborative project of the University of Crete, the Foundation for Research and Technology-Hellas and the Max-Planck-Institut für Extraterrestrische Physik. This work has been supported by a P.EN.E.D. program of the General Secretariat of Research and Technology of Greece.

\section{References}

Acker, 1978, A\&AS, 33, 367

Alexander, J., \& Balick, B. 1997, AJ, 114, 713

Aller, L. H. 1984, Physics of thermal gaseous nebulae (D. Reidel Publishing Company)

Bachiller, R., Huggins, P. J., Cox, P., \& Forveille, T. 1993, A\&A, 267, 177

Balick, B. 1987, AJ, 94, 671

Balick, B., Perinotto, M., Maccioni, A., Terzian, Y., \& Hajian, A. 1994, ApJ, 424, 800

Bohigas, J. 1994, A\&A, 288, 617

Bryce, M., Balick, B., \& Meaburn, J. 1994, MNRAS, 266, 721

Cardelli, J. A., Clayton, G. C., \& Mathis, J. S. 1989, ApJ, 345, 245

Chu, Y. H., Gacoby, G. H., \& Arendt, R. 1987, ApJS, 64, 529

Collins, G. W., Daub, C. T., \& O’Dell, C. R. 1961, ApJ, 133, 471

Corradi, R. L. M., \& Schwarz, H. E. 1995, A\&A, 293, 871

Corradi, R. L. M., Schönberner, D., Steffen, M., \& Perinotto, M. 2000, A\&A, 354, 1071

Corradi, R. L. M., Manso, R., Mampaso, A., \& Schwarz, H. E. 1996, A\&A, 313, 913

Frank, A., Balick, B., Icke, V., \& Mellema, G. 1993, ApJ, 404, L25

Frank, A. 1994, AJ, 107, 261

Frank, A., \& Mellema, G. 1994, ApJ, 430, 800

Górny, S. K., Stasińska, G., \& Tylenda, R. 1997, A\&A, 318, 256

Gathier, R., \& Pottasch, S. R. 1989, A\&A, 209, 369

Hummer, D. G., \& Storey, P. J. 1987, MNRAS, 224, 801

Kaler, J. B., Shaw, R. A., \& Kwitter, K. B. 1990, ApJ, 359, 392

Lame, N. J., \& Pogge, R. W. 1994, AJ, 108, 1860

Lame, N. J., \& Pogge, R. W. 1996, AJ, 111, 2320

Osterbrock, D. E. 1989, Astrophysics of gaseous nebulae (W. H. Freeman \& Company)

Schönberner, D. 1983, ApJ, 272, 708

Steffen, M., Szczerba, R., \& Schönberner, D. 1998, A\&A, 337, 149

Tylenda, R. 1986, A\&A, 156, 217

Zhang, C. Y., \& Kwok, S. 1998, ApJS, 117, 341

Zuckerman, B., Kastner, J. H., \& Gatley, I. 1990, ApJ, 356, L59 